\title{
A New Method of Image Denoising Based on the Energy Method
}

\author{
Shuang Liang, Shuli Wang, and Yu Wang
}

\begin{abstract}
To sove the minimization problem of the energy method in image denoising, the diffusion process of the Euler-Lagrange could be decomposed to the diffusions along the tangential direction and normal direction. In order to denoising and retained the texture information of the real scene as possible, at the texture pixels, the diffusion along the tangential direction should be retained and the diffusion along the normal direction should be controlled. Therefore we propose a new denoising method with edge diffusion along the tangential direction directly. The performance of this method is tested by single images in this thesis.
\end{abstract}

Index Terms-Image denoising, energy method, edge diffusion, tangential direction, normal direction.

\section{INTRODUCTION}

As for images in different scenes and with different kinds of noise type, many models have been studied in image denoising in the previous researches [1-30]. In this paper, we study the problem of image denoising based on the energy method [16], [21], [26]. Denote the real image as $u: \Omega \subset$ $R^{2} \rightarrow R$ and the degraded image as $u_{0}$. Then the degradation model of a digital image is

$$
u_{0}=R u+\eta
$$

where $R$ is a linear operator inducing image degradation for objective reasons, and $\eta$ refers to Gaussian White Noise in this paper.

Tikhonov and Arsenin proposed energy functional added a regular term

$$
E(u)=\int_{\Omega}\left|u_{0}-R u\right|^{2} d x+\lambda \int_{\Omega}|\nabla u|^{2} d x
$$

where the first term refers to the difference between the restored image and the degraded image, the second term means the total gradient modulus of the restored image and $\lambda$ is a weighting parameter[1]. In regularization energy functional, two basic condition of denoising have been concerned. Firstly, the basic structures between the restored image and the degraded image must be close enough. Secondly, the total gradient modulus of the restored image must be smaller than which of the degraded image. Based on the above two basic conditions, the energy model was established. As a result, the problem of denoising has

Manuscript received September 26, 2016; revised January 13, 2017.

Shuang Liang, Shuli Wang, and $\mathrm{Yu}$ Wang are with School of Mathematical Sciences, Peking University, Beijing (e-mail: liangshuang12@pku.edu.cn, wangyu_amo@pku.edu.cn). changed to the minimization problem of energy model

$$
\inf _{u}\left\{E(u), u \in W^{1,2}(\Omega)\right\}
$$

Furthermore, in Ref. [3] Aubert and Vese proposed energy functional added a smooth term

$$
E(u)=\int_{\Omega}\left|u_{0}-R u\right|^{2} d x+\lambda \int_{\Omega} \phi(|\nabla u|) d x .
$$

In order to solve the minimization problem, Chambolle proposed the projection approach in Ref. [6] and Lions proposed the half-quadratic minimization approach in Ref. [9]. These two methods both have better performance. In fact, in the previous researches, the Euler-Lagrange equation was also mentioned. The solution of the minimization problem also satisfied the Euler-Lagrange equation

$$
\left\{\begin{array}{ll}
R^{*} R u-R^{*} u_{0}-\lambda \Delta u=0 & \text { in } \Omega \\
\frac{\partial u}{\partial N}=0 & \text { on } \partial \Omega
\end{array} .\right.
$$

In Ref. [23], Perona and Marik noticed that the diffusion process of the Euler-Lagrange could be decomposed to the diffusions along the tangential direction and normal direction respectively, then proposed nonlinear diffusion partial differential equation

$$
\frac{\partial u}{\partial t}(t, x)=c\left(|\nabla u|^{2}\right) u_{T T}+b\left(|\nabla u|^{2}\right) u_{N N} .
$$

In order to weaken noise and retain the texture information of the real scene as possible, at the texture pixels, the diffusion along the tangential direction should be retained and the diffusion along the normal direction should be controlled. Based on the above analysis, we propose a new denoising method with edge diffusion along the tangential direction directly. The performance of this method is tested by single images in this thesis.

The rest of the paper is organized as follows. In Section II, the detail of the proposed denoising method is presented. The experiments results and comparisons are shown in Section III. Finally, the conclusion is given in Section IV.

\section{The PROPOSED METHOD}

Consider the energy functional added a smooth term

$$
E(u)=\int_{\Omega}\left|u_{0}-R u\right|^{2} d x+\lambda \int_{\Omega} \phi(|\nabla u|) d x
$$

Supposing $E(u)$ has a minimum at $u$, it must satisfy the 
Euler-Lagrange equation

$$
R^{*} R u-\lambda \operatorname{div}\left(\frac{\phi^{\prime}(|\nabla u|)}{|\nabla u|} \nabla u\right)=R^{*} u_{0},
$$

And it is a diffusion equation. For $x$ with $\nabla u(x) \neq 0$, define the unit normal vector of $x$ as $N(x)=\frac{\nabla u(x)}{|\nabla u(x)|}$ and the unit tangent vector of $x$ as $T(x),|T(x)|=1, T(x) \perp N(x)$. Take note of

$$
\begin{gathered}
u_{T T}=T^{t} \nabla^{2} u T=\frac{1}{|\nabla u|^{2}}\left(u_{x_{1}}^{2} u_{x_{2} x_{2}}+u_{x_{2}}^{2} u_{x_{1} x_{1}}-\right. \\
\left.2 u_{x_{1}} u_{x_{2}} u_{x_{1} x_{2}}\right), \\
u_{N N}=N^{t} \nabla^{2} u N=\frac{1}{|\nabla u|^{2}}\left(u_{x_{1}}^{2} u_{x_{1} x_{1}}+u_{x_{2}}^{2} u_{x_{2} x_{2}}+\right. \\
\left.2 u_{x_{1}} u_{x_{2}} u_{x_{1} x_{2}}\right) .
\end{gathered}
$$

where $u_{x_{1}} u_{x_{2}}$ refer to the first partial derivatives and $u_{x_{1} x_{1}}, u_{x_{1} x_{2}}, u_{x_{2} x_{2}}$ refer to the second partial derivatives. Then the Euler-Lagrange equation can be rewrite

$$
R^{*} R u-\lambda\left(\frac{\phi^{\prime}(|\nabla u|)}{|\nabla u|} u_{T T}+\phi^{\prime \prime}(|\nabla u|) u_{N N}\right)=R^{*} u_{0}
$$

The diffusion process of the Euler-Lagrange is decomposed to the diffusions along the tangential direction and normal direction. In order to denoising and retained the texture information of the real scene as possible, at the texture pixels, the diffusion along the tangential direction should be retained and the diffusion along the normal direction should be controlled. To come true isotropy in smooth area and the diffusion along the tangential direction, the function $\phi(s)$ should satisfy

$$
\begin{gathered}
\phi^{\prime}(0)=0, \phi^{\prime \prime}(0)>0 \\
\lim _{s \rightarrow+\infty} \phi^{\prime \prime}(s)=\lim _{s \rightarrow+\infty} \frac{\phi(s)}{s}=0, \lim _{s \rightarrow+\infty} \frac{\phi^{\prime \prime}(s)}{\phi(s) / s}
\end{gathered}
$$

Based on the above analysis, we propose a new denoising method with edge diffusion along the tangential direction directly as described below:

1) Preprocessing. To smooth the degraded image $I_{\text {in }}$ locally and denote the processed image as $S I_{\text {in }}$.

2) Calculate the gradient vector $\nabla S I_{\text {in }}$ and $\nabla^{\perp} S I_{\text {in }}$ perpendicular to $\nabla S I_{i n}$ at each point $(i, j)$ in $S I_{i n}$. Obviously, the unit normal vector is $N(i, j)=\frac{\nabla S I_{i n}(i, j)}{\left|\nabla S I_{i n}(i, j)\right|}$ and the unit tangent vector $T(i, j)=\frac{\nabla^{\perp} S I_{i n}(i, j)}{\left|\nabla^{\perp} S I_{i n}(i, j)\right|}$.

3) At each point $(i, j)$ in $S I_{i n}$, calculate the projections of $\nabla^{\perp} S I_{\text {in }}$ on the eight directions in nine points neighborhood $\nabla^{\perp} S I_{\text {in }} \cdot \overrightarrow{e_{k}}, k=1,2, \cdots, 8$, where

$$
\begin{gathered}
e_{1}=(1,0), e_{2}=\left(\frac{\sqrt{2}}{2}, \frac{\sqrt{2}}{2}\right), \\
e_{3}=(0,1), e_{4}=\left(-\frac{\sqrt{2}}{2}, \frac{\sqrt{2}}{2}\right),
\end{gathered}
$$

$$
\begin{gathered}
e_{5}=(-1,0), e_{6}=\left(-\frac{\sqrt{2}}{2},-\frac{\sqrt{2}}{2}\right), \\
e_{7}=(0,-1), e_{8}=\left(\frac{\sqrt{2}}{2},-\frac{\sqrt{2}}{2}\right) .
\end{gathered}
$$

4) Diffusion. Assign different weights as the following method

$$
\begin{aligned}
& S I_{i n}(i+1, j)+\lambda S I_{i n}(i, j) \cdot \frac{\left|\nabla^{\perp} S I_{i n} \cdot \overrightarrow{e_{1}}\right|}{\sum_{k=1}^{8}\left|\nabla^{\perp} S I_{i n} \cdot \overrightarrow{e_{k}}\right|+\varepsilon} \\
& \rightarrow S I_{\text {in }}(i+1, j) \text {, } \\
& S I_{i n}(i+1, j+1)+\lambda S I_{i n}(i, j) \cdot \frac{\left|\nabla^{\perp} S I_{i n} \cdot \overrightarrow{e_{2}}\right|}{\sum_{k=1}^{8}\left|\nabla^{\perp} S I_{i n} \cdot \overrightarrow{e_{k}}\right|+\varepsilon} \\
& \rightarrow S I_{i n}(i+1, j+1) \text {, } \\
& S I_{i n}(i, j+1)+\lambda S I_{i n}(i, j) \cdot \frac{\left|\nabla^{\perp} S I_{i n} \cdot \overrightarrow{e_{3}}\right|}{\sum_{k=1}^{8}\left|\nabla^{\perp} S I_{i n} \cdot \overrightarrow{e_{k}}\right|+\varepsilon} \\
& \rightarrow S I_{i n}(i, j+1) \text {, } \\
& S I_{i n}(i-1, j+1)+\lambda S I_{i n}(i, j) \cdot \frac{\left|\nabla^{\perp} S I_{i n} \cdot \overrightarrow{e_{4}}\right|}{\sum_{k=1}^{8}\left|\nabla^{\perp} S I_{i n} \cdot \overrightarrow{e_{k}}\right|+\varepsilon} \\
& \rightarrow S I_{i n}(i-1, j+1) \text {, } \\
& S I_{i n}(i-1, j)+\lambda S I_{i n}(i, j) \cdot \frac{\left|\nabla^{\perp} S I_{i n} \cdot \overrightarrow{e_{5}}\right|}{\sum_{k=1}^{8}\left|\nabla^{\perp} S I_{i n} \cdot \overrightarrow{e_{k}}\right|+\varepsilon} \\
& \rightarrow S I_{\text {in }}(i-1, j) \text {, } \\
& S I_{\text {in }}(i-1, j-1)+\lambda S I_{\text {in }}(i, j) \cdot \frac{\left|\nabla^{\perp} S I_{\text {in }} \cdot \overrightarrow{e_{6}}\right|}{\sum_{k=1}^{8}\left|\nabla^{\perp} S I_{\text {in }} \cdot \overrightarrow{e_{k}}\right|+\varepsilon} \\
& \rightarrow S I_{i n}(i-1, j-1) \text {, } \\
& S I_{i n}(i, j-1)+\lambda S I_{i n}(i, j) \cdot \frac{\left|\nabla^{\perp} S I_{i n} \cdot \overrightarrow{e_{7}}\right|}{\sum_{k=1}^{8}\left|\nabla^{\perp} S I_{i n} \cdot \overrightarrow{e_{k}}\right|+\varepsilon} \\
& \rightarrow S I_{i n}(i, j-1) \text {, } \\
& S I_{i n}(i+1, j-1)+\lambda S I_{i n}(i, j) \cdot \frac{\left|\nabla^{\perp} S I_{i n} \cdot \overrightarrow{e_{11}}\right|}{\sum_{k=1}^{8}\left|\nabla^{\perp} S I_{i n} \cdot \overrightarrow{e_{k}}\right|+\varepsilon}
\end{aligned}
$$

where $\lambda>0,0<\varepsilon \ll 1$.

5) The image gray value increase after the process of diffusion. So an appropriate method to make the gray value belonging in $[0,255]$ is necessary. Here we use histogram matching method and average gray value translation method, thus keep the image average gray value and image histogram unchanged essentially.

6) Iteration. Repeat the above steps 1) - 5) if the image denoising result is unsatisfied.

\section{EXPERIMENTAL RESULTS}

To evaluate the performance of our method, we have tested it on some test images shown in Fig.1 - Fig.3. In the experiment, we add noise of Gaussian $(0,0.05)$ on each test image and observe denoising results of the first iteration and the next one applying our method. Furthermore, we measure the quality of the denoising images with some methods of image quality assessment, i.e. MSE, PSNR, SSIM, MSSIM, SNR, UQI, and VIFP, as is shown in Table I - Table III. 

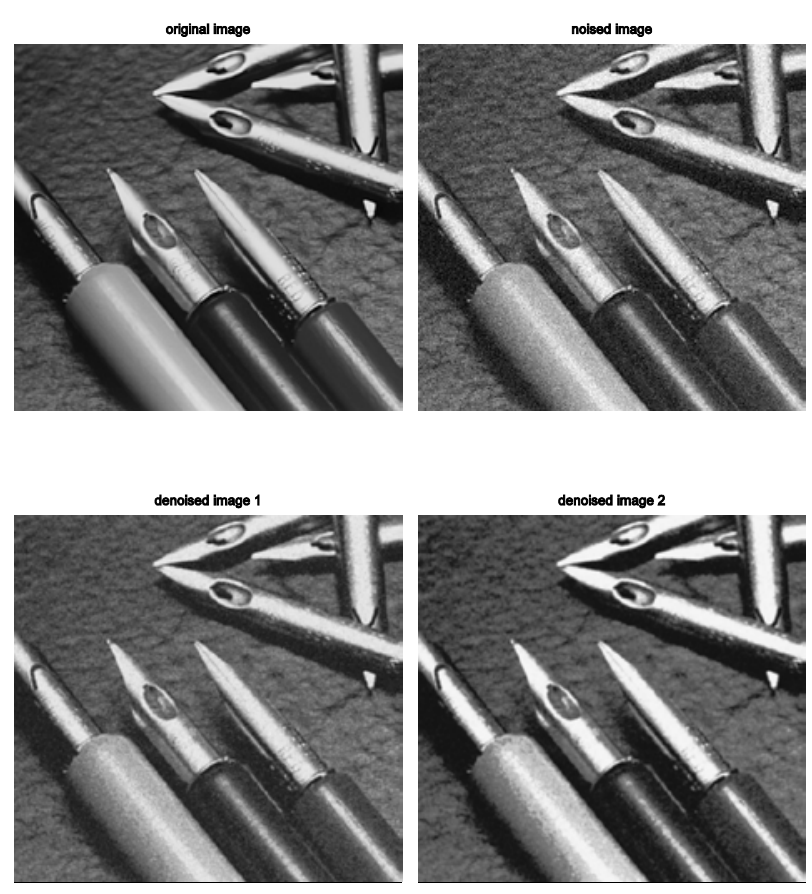

Fig. 1. Pens.

\begin{tabular}{|c|c|c|c|c|c|}
\hline \multirow{2}{*}{$\begin{array}{l}\text { Methods } \\
\text { of image } \\
\text { quality } \\
\text { assess- } \\
\text { ment }\end{array}$} & & $\begin{array}{l}\text { Denoising } \\
\text { the first i }\end{array}$ & $\begin{array}{l}\text { esults of } \\
\text { ration }\end{array}$ & \multicolumn{2}{|c|}{$\begin{array}{l}\text { Denoising results of } \\
\text { the second iteration }\end{array}$} \\
\hline & $\begin{array}{l}\text { noising } \\
\text { image }\end{array}$ & $\begin{array}{l}\text { denoisin } \\
\text { g } \\
\text { image }\end{array}$ & $\begin{array}{l}\text { optimi } \\
\text {-ed } \\
\text { rate(\% } \\
\text { ) }\end{array}$ & $\begin{array}{l}\text { denoisin } \\
\text { g } \\
\text { image }\end{array}$ & $\begin{array}{l}\text { optimi- } \\
\text { ed } \\
\text { rate(\%) }\end{array}$ \\
\hline MSE & 781.3902 & 233.8726 & 70.07 & 232.1473 & 70.29 \\
\hline PSNR & 19.2021 & 24.4410 & 27.28 & 24.4732 & 27.45 \\
\hline SSIM & 0.3044 & 0.8347 & 174.18 & 0.8628 & 183.43 \\
\hline MSSIM & 0.8194 & 0.9211 & 12.41 & 0.9221 & 12.53 \\
\hline SNR & 11.7809 & 17.0198 & 44.47 & 17.0520 & 44.74 \\
\hline UQI & 0.3212 & 0.6184 & 92.53 & 0.6532 & 103.37 \\
\hline VIFP & 0.2232 & 0.3714 & 66.40 & 0.3893 & 74.41 \\
\hline
\end{tabular}
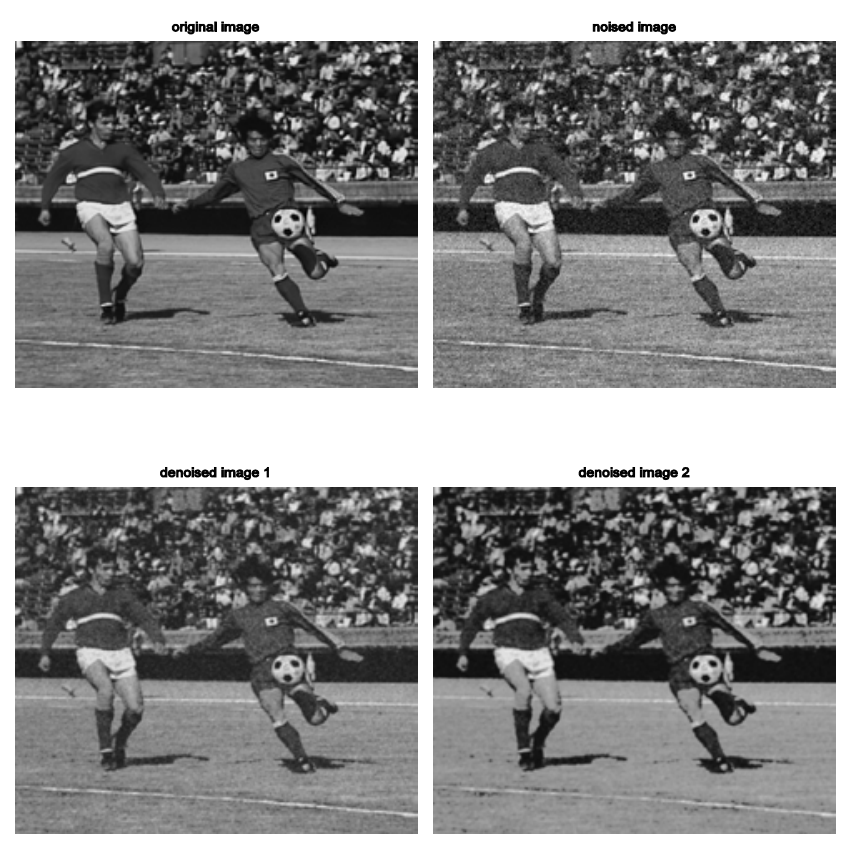

Fig. 2. Soccer.

\begin{tabular}{|c|c|c|c|c|c|}
\hline \multirow{2}{*}{$\begin{array}{c}\text { Methods } \\
\text { of image } \\
\text { quality } \\
\text { assess- } \\
\text { ment } \\
\end{array}$} & & \multicolumn{2}{|c|}{$\begin{array}{l}\text { Denoising results of } \\
\text { the first iteration }\end{array}$} & \multicolumn{2}{|c|}{$\begin{array}{l}\text { Denoising results of } \\
\text { the second iteration }\end{array}$} \\
\hline & $\begin{array}{l}\text { noising } \\
\text { image }\end{array}$ & $\begin{array}{l}\text { denoising } \\
\text { image }\end{array}$ & $\begin{array}{l}\text { optimiz- } \\
\text { ed } \\
\text { rate(\%) }\end{array}$ & $\begin{array}{l}\text { denoising } \\
\text { image }\end{array}$ & $\begin{array}{l}\text { optimiz- } \\
\text { ed } \\
\text { rate(\%) }\end{array}$ \\
\hline MSE & 792.9716 & 294.0847 & 62.91 & 387.7826 & 51.10 \\
\hline PSNR & 19.1382 & 23.4461 & 22.51 & 22.2449 & 16.23 \\
\hline SSIM & 0.4337 & 0.8546 & 97.06 & 0.8350 & 92.54 \\
\hline MSSIM & 0.8684 & 0.9282 & 6.89 & 0.8989 & 3.51 \\
\hline SNR & 11.5662 & 15.8740 & 37.25 & 14.6729 & 26.86 \\
\hline UQI & 0.4662 & 0.7009 & 50.33 & 0.6527 & 40.01 \\
\hline VIFP & 0.2455 & 0.3771 & 53.64 & 0.3371 & 37.31 \\
\hline
\end{tabular}
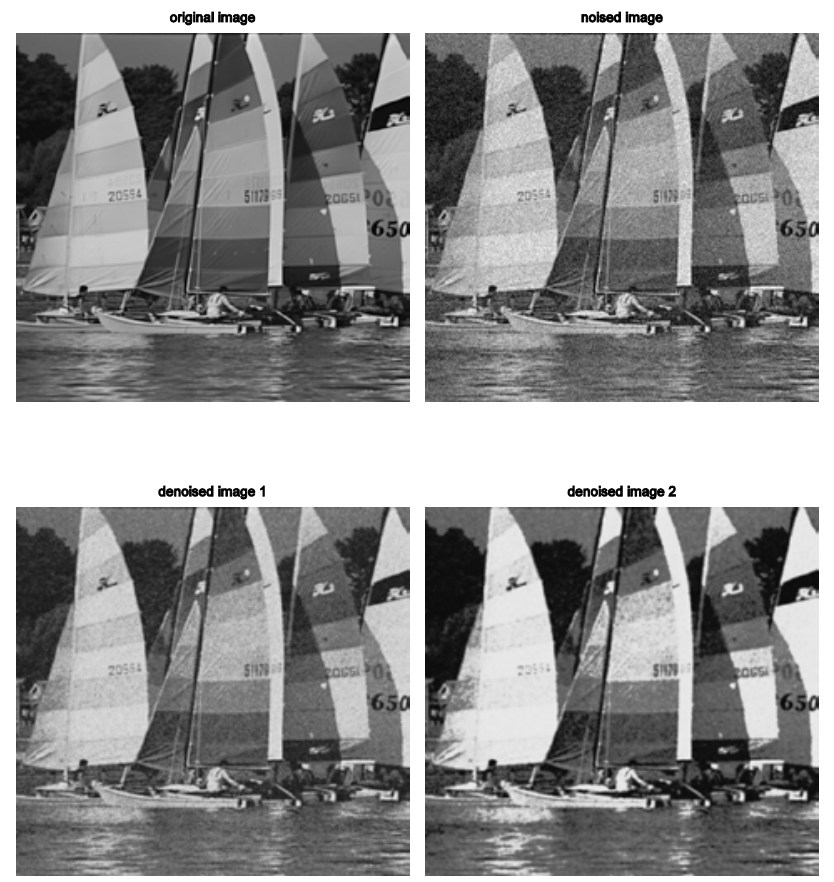

Fig. 3. Yach.

\begin{tabular}{|c|c|c|c|c|c|}
\hline $\begin{array}{c}\text { Methods } \\
\text { of image } \\
\text { quality } \\
\text { assess- } \\
\text { ment }\end{array}$ & \multicolumn{4}{|c|}{$\begin{array}{c}\text { TABLE III: YACH } \\
\text { denoising results of } \\
\text { the first iteration }\end{array}$} & $\begin{array}{l}\text { denoising results of } \\
\text { the second iteration }\end{array}$ \\
\hline & $\begin{array}{c}\text { noising } \\
\text { image }\end{array}$ & $\begin{array}{c}\text { denoising } \\
\text { image }\end{array}$ & $\begin{array}{l}\text { optimiz- } \\
\text { ed } \\
\text { rate(\%) }\end{array}$ & $\begin{array}{l}\text { denoising } \\
\text { image }\end{array}$ & $\begin{array}{l}\text { optimiz- } \\
\text { ed } \\
\text { rate(\%) }\end{array}$ \\
\hline MSE & 797.4814 & 305.7834 & 61.66 & 727.1404 & 8.82 \\
\hline PSNR & 19.1136 & 23.2767 & 21.78 & 19.5146 & 2.10 \\
\hline SSIM & 0.3438 & 0.8190 & 138.24 & 0.8130 & 136.51 \\
\hline MSSIM & 0.8126 & 0.9074 & 11.67 & 0.8746 & 7.63 \\
\hline SNR & 12.5901 & 16.7531 & 33.07 & 12.9911 & 3.19 \\
\hline UQI & 0.3449 & 0.5482 & 58.93 & 0.5297 & 53.58 \\
\hline VIFP & 0.2242 & 0.3422 & 52.64 & 0.3285 & 46.53 \\
\hline
\end{tabular}

\section{CONCLUSION}

In this paper, we study the problem of image denoising based on the energy method. Based on the previous research, we propose a new denoising method with edge diffusion along the tangential direction directly. The performance of 
this method is tested by single images in this thesis.

\section{REFERENCES}

[1] A. N. Tikhonov and V. Y. Arsenin, Solutions of Ill-posed Problem, Halsted Press, New York, 1977.

[2] L. Rudin, S. Osher, and E. Fatemi, "Nonlinear total variation based noise removal algorithm,” Physica D, 60, pp. 259-268, 1992.

[3] G. Auber and L. Vese, "A variational method in image recovery," SIAM Journal of Numerical Analysis, vol. 34, no. 5, pp. 1948-1979, 1997.

[4] P. Kornprobst, R. Deriche, and G. Aubert, "Nonlinear operators in image restoration," in Pro. of the international Conference of Computer Vision and Pattern Recognition, pp. 325-331, Puerto Rico, June 1997.

[5] L. Vese, "A study in the BV space of a denoising-deblurring variational problem,” Applied Mathematics and Optimization, vol. 44, no. 2, pp. 131-161, 2001.

[6] A. Chambolle, "An algorithm for total variation minimization and applications,” Journal of Mathematical Imaging and Vision, vol. 20, no. 1-2, pp. 89-97, March 2004.

[7] A. Braides, "Gamma-convergence for beginners," In Oxford Lecture Series in Math in Mathematics and Its Applications, no. 22, Oxford University Press, 2002.

[8] J. B. H. Urruty and C. Lemar'echal, "Convex analysis and minimization algorithms,” Grundlehrender Mathematischen Wissenschafen [Fundamental Principles of Mathematical Sciences], Springer-Verlag: Berlin, 1993.

[9] A. Chambolle and P. L. Lions, "Image recovery via total variation minimization and related problem,” Numerische Mathematic, vol. 76 no. 2, pp. 167-1188, 1997.

[10] F. Demengel and R. Teman, "Convex functions of a measure and applications,” Indiana University Mathematics Journal, vol. 33, pp. 673-709, 1984.

[11] A. Kirsch, "An introduction to the mathematical theory of inverse problems,” Applied Mathematical Sciences, Spirnger, vol. 120, 1996.

[12] M. Bertero and P. Boccacci, "Introduction to inverse problem in imaging,” Bristol: IoP, Institute of Physics Publishing, 1998.

[13] C. Bouman and K. Sauer, "A generalized gaussian model for edge-preserving MAP estimation,” IEEE Transactions on Image Processing, vol. 2, no. 3, pp. 296-310, 1993.

[14] L. Vese, "Problems variationels et EDP pour 1'analyse d'images et 1'evolution de courbes," $\mathrm{PhD}$ thesis, University de Nice Sophia-Antipolis, Nov 1996.

[15] L. Vese, "A study in the BV space of a denoising-deblurring variational problem,” Applied Mathmaticals and Optimization, vol. 44, no. 2, pp. 131-161, 2001.

[16] L. Vese and S. Osher, "Modeling textures with total variation minimization and oscillating patterns in image processing," Journal of Scientific Computing, vol. 19, pp. 553-572, 2003.

[17] D. Geman and G. Reynolds, "Constrained restoration and the recovery of discontinuities," IEEE Trans. On Pattern Analysis and Machine Intelligence, vol. 14, no. 3, pp. 367-383, 1993.

[18] P. Charbonnier, G. Aubert, M. Blanc-Feraud, and M. Barlaud, “Two deterministic half quadratic regularization algorithms for computed imaging,” in Pro. the International Conference on Image Processing, vol. II, pp. 168-172,1994

[19] L. Rudin, S. Osher, and E. Fatemi, "Nonlinear total variation based noise removal algorithms,” Physica D, vol. 60, pp. 259-268,1992.

[20] R. T. Rockafellar, Convex Analysis, Princeton University Press, Princeton, N. J., 1970.

[21] J. Bect, L. B. Feraud, G. Aubert, and A. Chambolle, “A $l^{1}$-unified variational framework for image restoration," in Proc. the 8th European Conference on Computer Vision, vol. IV, T. Pajdla and J. Matas, Ed. Prague, Czach Republic, 2004.
[22] L. C. Evan, "Partial differential equations, vol. 19 of graduate studies in mathematics," Proceeding of the America Mathmatical Society, 1998.

[23] P. Perona and J. Malik," Scale-space and edge detection using anisotropoc diffusion,” IEEE Trans. on Pattern Analysis and Machine Intelligence, vol. 12, no. 7, pp. 629-639, July 1990.

[24] J. Weickert, "Theoretial foundations of anisotropic diffusion in image processing,” Computing Supplement, vol. 11, pp. 221-236, 1996.

[25] J. Weickert, "Coherence-enhancing diffusion filtering," The International Journal of Computer Vision, vol. 31m, no. 2/3, pp. 111-127, April 1999

[26] G. Aubert and P. Kronprobst, Mathematical Problems in Image Processing: Partial Differential Equations and the Calculus of Variarions, 2nd ed., Springer, October 2009.

[27] A. Chambolle and G. Dal Maso, "Discrete approximations of the Mumford-Shah functional in dimension two,” M2AN, vol. 33, no. 4, pp. 651-672, 1999.

[28] T. Chan and J. Shen, "Mathematical models for local non-texture inpaintings," SIAM Journal of Applied Mathematics, vol. 62, pp. 1019-1043, 2002.

[29] T. Chan and L. Vese, "A level set algorithm for minimizing the Mumford-Shah functional in image processing," in Proc. 1st IEEE Workshop on Vairational and Level Set Methods in Computer Vision, pp. 161-168, 2001.

[30] T. Chan and J. Shen, "Image processing and analysis - variational, PDE, wavelet, and stochastic methods," Image Process, vol. 10, no. 5 , pp. 767-782, May 2001.

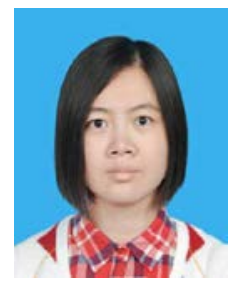

Shuang Liang was born in china at 1986. She received her bachelor's degree in fundamental and applied mathematics from Heibei Normal University in 2008, and her master's degree in applied mathematics from Heibei Normal University in 2011. She is currently pursuing her Ph.D. degree in applied mathematics from Beijing Peking University, China.

Her research interests include digital image processing, image quality assessment, and contourlet/wavelet transform theory, Etc. She has published around 10 scientific papers in journals and conferences on digital image processing, image quality assessment, video signal processing, Etc.

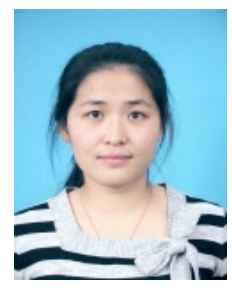

Shuli Wang was born in china at 1987. She received her bachelor's degree in fundamental and applied mathematics from Heibei Normal University in 2010 and her master's degree in applied mathematics from Heibei Normal University in 2013. She is currently pursuing her Ph.D. degree in applied mathematics from Beijing Peking University, China.

Her research interests include digital image processing, and wavelet transform theory, Etc

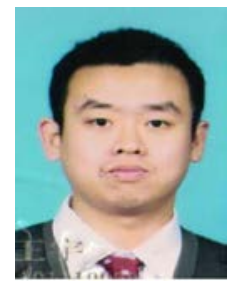

Shuang Liang was born in china at 1985 . He received his Ph.D. degree in applied mathematics from Beijing Peking University, China in 2015.

His research interests include digital image processing, image quality assessment Etc. He has published around 4 scientific papers in journals on digital image processing, image quality assessment, video signal processing, Etc. 